\title{
Observation of a charged incoherent $\mathrm{BiFeO}_{3} / \mathrm{SrTiO}_{3}$ interface
}

\author{
Christopher Addiego ${ }^{1}$, Dianxiang $\mathrm{Ji}^{2}$ and Xiaoqing Pan $^{3}$ \\ ${ }^{1}$ University of California - Irvine, IRVINE, California, United States, ${ }^{2}$ The Hong Kong Polytechnic University, \\ United States, ${ }^{3}$ Department of Physics and Astronomy, University of California, Irvine, CA 92697, Irvine, \\ California, United States
}

Emergent phenomena often occur at oxide heterostructures due to strong coupling between their available degrees of freedom. In ferroelectric oxides, the lattice, charge, and polarization are often coupled and can lead to localized regions with unique electrical properties [1]. This can result in the formation of 2D free carrier gases forming at the interface with properties that are modulated by the local polarization. For example, at a $\mathrm{BiFeO}_{3} / \mathrm{TbScO}_{3}$ interface, it was found that the polarization direction could switch the carrier type at the interface [2]. However, most of the interfaces that have been studied were coherent, with perfect epitaxy between the substrate and thin film. In perovskite oxides $\left(\mathrm{ABO}_{3}\right)$ structures, this typically means that the $\mathrm{A} / \mathrm{B}$ sites of the substrate are aligned with the $\mathrm{A} / \mathrm{B}$ sites of the thin film. But with advances in thin film growth methods, more complex interfaces can also be synthesized. In this work, we synthesized a $\mathrm{BiFeO}_{3}(\mathrm{BFO})$ thin film on an $\mathrm{SrTiO}_{3}$ (STO) substrate with an incoherent interface where the BFO and STO unit cells are misaligned by a half step along [100] direction.

At the interface, we observe a rock-salt like $\mathrm{BiO}$ double-layer with interstitial doping. For comparison, HAADF images of the coherent and incoherent interface are shown in Figures 1a and 1b, respectively. This structure is similar to that found in previous work on $\mathrm{Bi}_{2} \mathrm{FeMnO}_{6} / \mathrm{STO}$ interfaces $[3,4]$. In the film with the coherent interface, the polarization in BFO points along the diagonal which would correspond to the rhombohedral-like phase. In the film with the incoherent interface, the polarization also points along the diagonal in the bulk region, but the last unit cell of BFO above the interface shows rotation towards [001] which would indicate a shift to the tetragonal-like phase and the first unit cell of STO below the interface also shows slight polarization. The c/a ratio across the interface in both cases is shown in Figure 1c. In the coherent interface, the c/a ratio increases from just below 1 in STO to $\sim 1.05$ in bulk BFO, as expected due to the change from the cubic to monoclinic structures. However, for the incoherent interface, the c/a ratio spikes to greater than 1.12 in the unit cells on either side of the interface, indicating a strong tetragonal distortion.

With the polarization pointing towards the incoherent interface from both sides, this is a strong indication that there may be charge accumulating in the region. Therefore, we performed atomic resolution four-dimensional scanning transmission electron microscopy (4D STEM) to investigate the local charge distribution. A reconstructed ADF image of the interface is shown in Figure 2a and the associated charge density is shown in Figure $2 \mathrm{~b}$. The charge density map reveals the full structure of the interfacial region, including $\mathrm{O}$ columns and Fe dopants. As previously demonstrated, integrating the charge according to Bader charge analysis gives an indication of the charge state of each atomic species [5]. We applied this analysis to all Bi sites in the BFO film and interfacial layer and also the $\mathrm{Sr}$ sites in STO. The average charge state of each layer is plotted across the interface in Fig. 2c. Sr sites in STO show a consistent charge state between 0.5-1e while Bi sites in BFO show a charge state > 1.2e. However, Bi sites inside the interfacial phase, show a charge state of $0.76 \mathrm{e}$ in the lower layer and $0.88 \mathrm{e}$ in the upper layer. The reduced charge state of $\mathrm{Bi}$ in the lower layer is an indication of the accumulation of free electrons at the interface. 


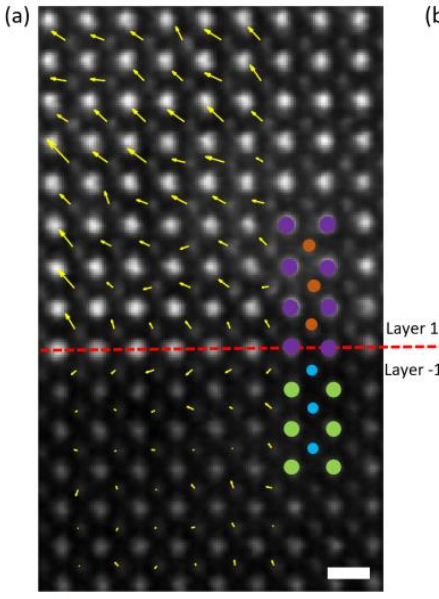

$\bullet \mathrm{Bi} \bullet \mathrm{Fe} \bullet \mathrm{Sr} \bullet \mathrm{Ti}$ (b)

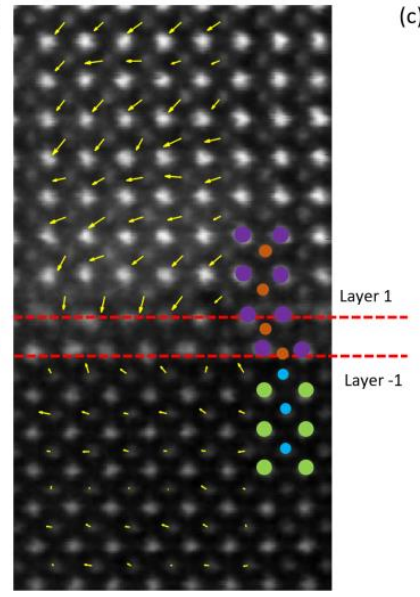

(c)

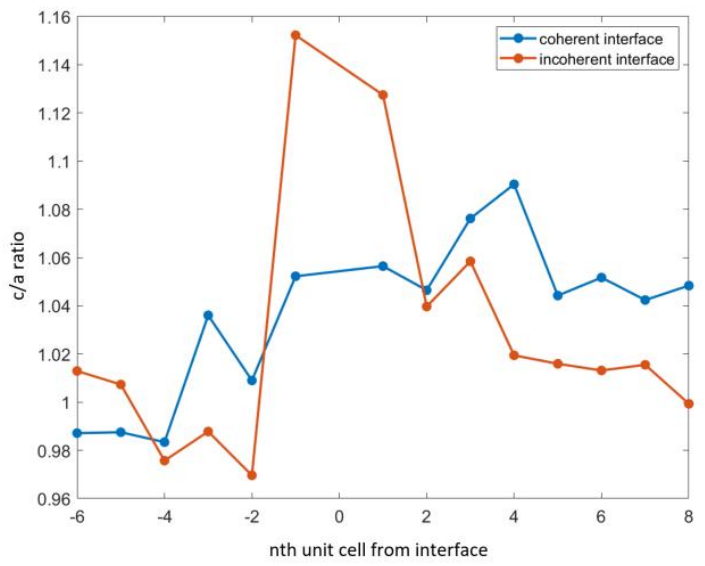

Figure 1. Fig 1. (a, b) HAADF image of a coherent and an incoherent BFO/STO interface, respectively, with polarization maps and structure models overlaid. (c) The c/a ratio across both interfaces. The scale bar is $4 \AA$.

(a)

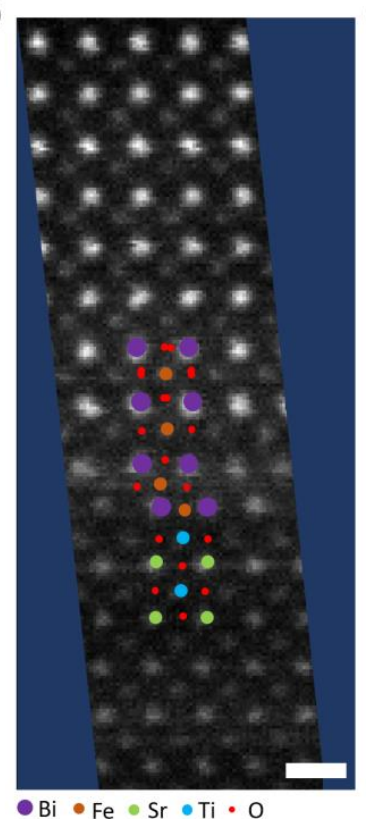

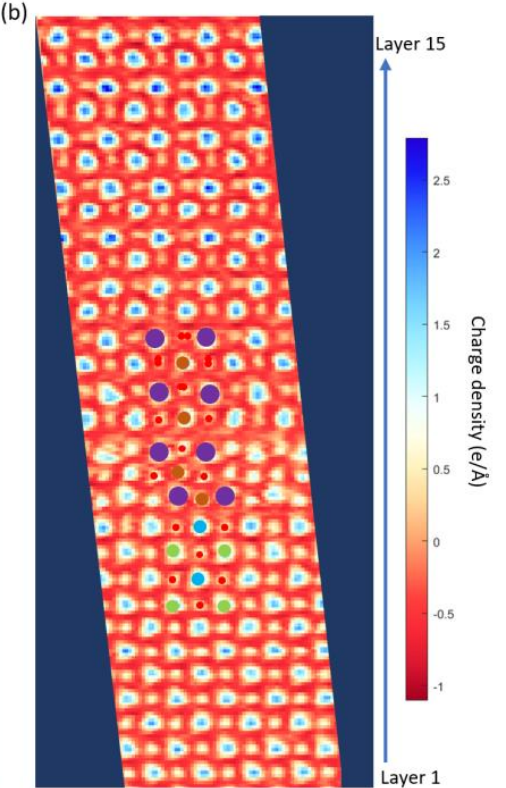

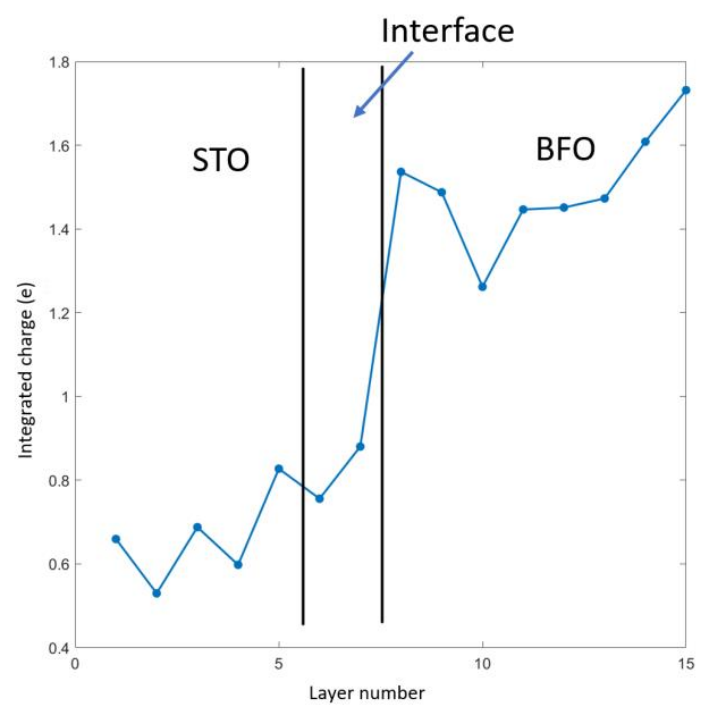

Figure 2. Fig 2. (a) An ADF image of the incoherent interface reconstructed from 4D STEM data. (b) The associated charge density. (c) The integrated charge of Sr and Bi sites across the interface. The scale bar is $4 \AA$.

\section{References}

[1] J. Manhart, et al., Science 26 (2010), 1607-1611.

[2] Y. Zhang et al., Nature Nanotechnology 13 (2018), 1132-1136.

[3] A.P. Chen et al., Advanced Materials 25 (2013), 1028-1032.

[4] Y. Zhu et al., APL Materials 1 (2013), 050702.

[6] W. Gao et al., Nature 575 (2019), 480-484.

[7] This work was supported by the Department of Energy (DOE) under Grant DE-SC0014430. The authors acknowledge the use of facilities and instrumentation at the UC Irvine Materials Research Institute (IMRI) supported in part by the National Science Foundation Materials Research Science and Engineering Center program through the UC Irvine Center for Complex and Active Materials (DMR-2011967). 